\title{
Iannis Xenakis et la musique française, une filiation incomprise?
}

\author{
Iannis Xenakis in francoska glasba, \\ nerazumljiva povezava?
}

Ključne besede: Iannis Xenakis, francoska glasba, Olivier Messiaen, François-Bernard Mâche, Pascal Dusapin

POVZETEK

Iannis Xenakis je v Franciji preživel vse svoje ustvarjalno obdobje. Brez dvoma je soočenje grškega duha in francoske glasbene kulture tisto, kar je izoblikovalo skladateljevo prepoznavno edinstvenost. Analiza tega soočenja od časa šolanja, še posebej, ko je spoznal Messaena, do poučevanja $\mathrm{v}$ zadnjih letih pa nam pravzaprav ostvetljuje bistvene sestavine francoske glasbe.
Mots clefs: Iannis Xenakis, musique française, Olivier Messiaen, François-Bernard Mâche, Pascal Dusapin

\section{RESUMÉE}

Iannis Xenakis a vécu en France l'intégralité de sa vie créatrice. C'est sans doute à la confrontation entre l'esprit grec et la culture musicale française que l'on doit la singularité lumineuse du compositeur. Depuis la période de formation, et en particulier la rencontre avec Messiaen, jusqu'à l'enseignement des dernières années, l'analyse de cette confrontation nous éclaire en retour sur des composantes essentielle de la musique française.

"Avec Xenakis, on se trouve au delà de l'histoire de la musique [...] Et on se demande (avec une certaine angoisse même) quelle est la nécessité (le sens le plus profond de cette nécessité) qui a conduit Xenakis à prendre radicalement parti de la sonorité 'objective' du monde contre celle d'une âme et de sa subjectivité sentimentale. ${ }^{1}$ "

On ne peut pas dire que l'importance d'un compositeur doive se mesurer à la fabrication de ses épigones. Dans ce sens, Pierre Boulez ou plus récemment Gérard Grisey, auront certainement eu plus d' 'aura', en France, que Iannis Xenakis. Non seulement Xenakis a toujours refusé d'enseigner la composition (de quelle tradition musicale aurait-

1 Milan Kundera, in Regards sur Iannis Xenakis, Paris Stock, 1981, p. 24. 
il pu véritablement se revendiquer?), mais il n'a jamais donné aux institutions qu'il a fondées un poids institutionnel qui en fasse des viviers de futurs clones musicaux. D'autre part, des compositeurs comme Francisco Guerrero en Espagne, ou Julio Estrada au Mexique sont probablement bien plus à même de se revendiquer de la pensée de Xenakis que n'importe quel compositeur français.

Xenakis est d'abord un compositeur $\mathrm{Grec}^{2}$, même s'il acquiert la nationalité française en $1965^{3}$. Et on ne doit sa venue en France qu'aux circonstańces historiques assez troubles qui ont marqué sa vie, à la fin de la deuxième guerre mondiale. Condamné à mort dans son pays, il fuit par l'Italie puis par la France en direction des États-Unis, où vivait son frère. Mais sa qualité de communiste dans la résistance grecque ne rend pas ce pays particulièrement indiqué. L'entraide de la communauté grecque à Paris, qui lui trouvera un emploi d'ingénieur, sera déterminante.

Rien n'est très évident, dans la place que prend la figure de Xenakis dans le monde de la composition en France, et sa mort, précédée de peu par une rétrospective bien timide au cours d'un festival Présence à la maison de Radio-France, ainsi que par un colloque organisé avec beaucoup d'énergie par Makis Solomos et le CDMC, n'a certes pas laissé le monde musical indifférent, mais a surtout révélé la distance prise par la musique française actuelle (du moins celle qui tient le haut du pavé médiatique et institutionnel) avec une des figures musicales les plus marquantes du vingtième siècle, si ce n'est, à mon sens, la plus marquante. Car Xenakis pourrait bien se révéler être, dans les années qui viennent, le repère d'exigence créative et intellectuelle à l'aune duquel nombre de productions musicales dont on nous rabat aujourd'hui les oreilles paraîtront manquer cruellement de sel.

Avant d'analyser plus avant cette situation paradoxale de la figure de Xenakis aujourd'hui en France, il convient de comprendre un peu mieux comment cet émigré grec a pu rencontrer dans le Paris du milieu des années cinquante un contexte intellectuel et artistique suffisamment stimulant pour lui suggérer une œuvre aussi forte. Outre la rencontre avec Le Corbusier, qui l'embauche comme ingénieur dans son cabinet d'architecture, on ne peut faire l'impasse ni sur la rencontre avec Olivier Messiaen, ni sur celle avec Pierre Schaeffer et le GRM, et, bien-sûr, sur les relations qu'il a eues avec Edgar Varèse.

La figure de Le Corbusier est certainement déterminante pour comprendre l'engagement de Xenakis du côté de la modernité. Même si les relations entre les deux hommes se sont détériorées après le conflit de paternité à propos du pavillon Philips à l'exposition universelle de Bruxelles en 1958, il est probable que l'on doit à l'architecte visionnaire une part non négligeable de l'intérêt de Xenakis pour le calcul des structures et les fonctionnalités de l'espace. Au delà de l'aspect alimentaire (assez médiocre du reste) de

2 "Il est très difficile à définir. Premièrement il est Grec - il n'y a rien à faire cette lucidité d'esprit, cette rapidité... "Si vous regardez le grand théâtre antique d'Eschyle, de Sophocle et d'Euripide, c'étaient des gens prodigieusement intelligents mais c'étaient des sujets horribles. C'est affreux! Ce sont des crimes épouvantables ! Il y a une certaine sauvagerie, et il y a un peu de ça chez lui. Il y a une certaine cruauté... oui.

"Finalement, ce qu'il a fait: il s'est servi des mathématiques, il s'est servi d'architecture, pour composer, et ça a donné quelque chose qui est tout à fait génial, mais qui est tout à fait en dehors. Qui n'est qu'à lui. Que personne d'autre ne pouvait faire! Ça a un impact, une force. Ça a une puissance."Olivier Messiaen, cité par Nouritza Matossian, Iannis Xenakis, Paris, Fayard/ Sacem, 1981, p. 5.

3 Gérard Condé, L'étoile Iannis Xenakis a rejoint sa galaxie, Paris, le Monde, mardi 6 février 2001, p. 35. 
son travail, le contact avec Le Corbusier lui aura permis de situer son œuvre dans le grand mouvement de conquête de l'espace personnel par les outils de la raison qui constitue le fer de lance de la pensée moderne dans son application à l'architecture. Au delà, il y trouvera aussi sans doute un sens de la dimension, une certaine audace, et l'assurance que la nouveauté d'une proposition vaut toujours mieux que le confort d'une idée toute faite. Le Corbusier avait aussi dédaigné les études traditionnelles d'architecture aux Beaux-Arts, au profit d'une formation plus scientifique.

Mais Xenakis n'est architecte que par nécessité et par hasard. Sa vocation la plus profonde le tourne vers la musique. Ses études dans ce domaine ont été, par la force des choses, un peu chaotiques. Il tente d'abord en vain de reprendre des études 'ordinaires' avec des professeurs aussi importants que Arthur Honegger ou Darius Milhaud. Sa première entrevue avec Honegger se cristallise autour de l'emploi des quintes et des octaves parallèles, qu'il revendique, ce qui lui vaut la fureur du maître. Ses rapports avec Milhaud ne sont guère plus enthousiastes. ${ }^{4}$ C'est alors qu'il fait la connaissance, en 1951, d'Olivier Messiaen au Conservatoire de Paris. La personnalité de Messiaen est unique dans le paysage musical français. Et sa manière de réagir, sa douceur et son ouverture d'esprit, a été pour Xenakis un véritable retournement. Messiaen comprend immédiatement qu'il a affaire à une personnalité hors du commun, que Xenakis est trop âgé (il a déjà trente ans) pour des études traditionnelles, et il l'encourage à être lui-même, c'est à dire grec, mathématicien et architecte, dans sa musique.

Messiaen n'a pas cherché à être un 'maître' pour Xenakis. Il lui a donné quelque chose de plus essentiel peut-être pour un artiste: un sentiment de confiance en soi. Il y a eu certainement entre Xenakis et Messiaen une forme de fascination réciproque que l'on peut entendre et comprendre dans la musique de chacun d'entre eux. Ainsi, certains passages particulièrement denses et complexes de Chronochromie (1960) sont directement sous l'influence de la combinatoire sérielle en vigueur à l'époque (Boulez fut également élève de Messiaen) mais aussi de modèles naturels peut-être plus 'stochastiques'. ' [...] la Nature, trésor inépuisable des couleurs et des sons, des formes et des rythmes, modèle inégalé de développement total et de variation perpétuelle [...]' déclare Messiaen à l'exposition universelle de Bruxelles en 1958! Dans l'autre sens, si l'on ne peut pas noter une influence musicale directe de Messiaen sur le tout premier Xenakis, on peut se demander jusqu'à quel point les recherches de Xenakis sur la théorie des cribles à partir de 1960 ne sont pas le prolongement des recherches de Messiaen sur les échelles ('modes') à transpositions limitées. L'emploi du Pelog javanais par Xenakis n'est peut-être pas étranger à l'attrait des musiciens français pour une certaine forme d'exotisme musical. Dans une' œuvre comme Tetora pour quatuor à cordes (1990), l'influence de Messiaen me paraît particulièrement audible, laissée à découvert, dans des zones où l'ambitus se restreint, par l'insistance sur des intervalles fétiches de Messiaen, comme la quinte diminuée descendante par exemple, et par des harmonisations parallèles elles aussi caractéristiques de la conception française (depuis Debussy) du rapport harmonie/timbre.

4 Cf. Nouritza Matossian, Iannis Xenakis, Paris, Fayard/Sacem, 1981, p. 41. 

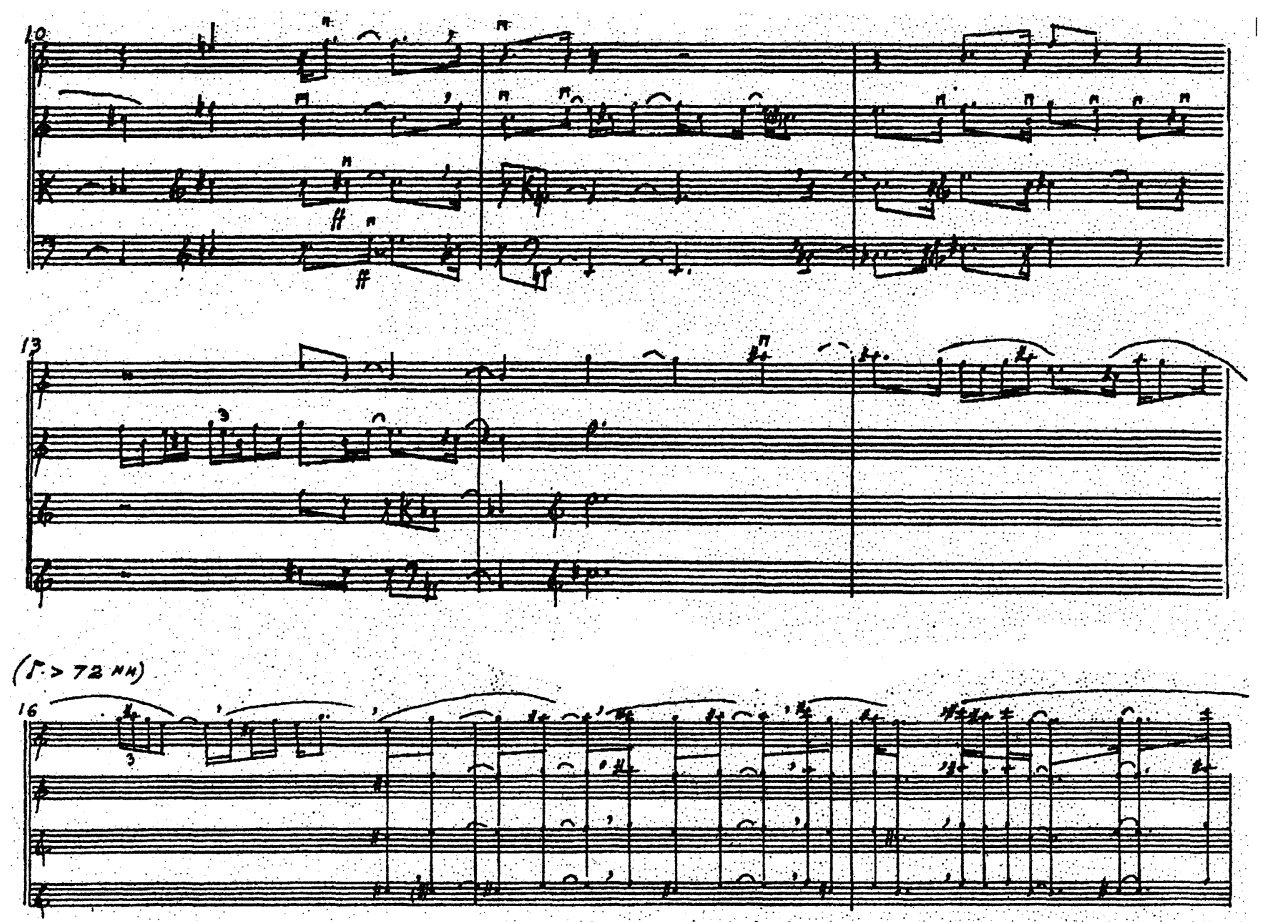

Ex. 1: extrait de Tetora de Iannis Xenakis pour Quatuor à corde (1990), M. 10-18 (avec l'aimable autorisation des édition Solabért, Paris).

D'une manière générale, les dernières œuvres de Xenakis sont marquées par ce souci de la complétude harmonique et les couleurs développées par cette complexité (par exemple dans Ioolkos pour orchestre de 1996) ne sont pas sans évoquer les accords couleur de 12 sons de Messiaen (Couleurs de la cité céleste...).

Ce sera aussi Messiaen qui va introduire Xenakis auprès de Pierre Schaeffer:

"Je vous recommande très spécialement mon élève et ami Iannis Xenakis, qui est Grec et très extraordinairement doué pour la musique et le rythme [...] D'autre part, il est désireux de faire de la musique concrète. Il pourrait devenir un de vos précieux collaborateurs. . ${ }^{5}$

Il réalisera au studio de musique concrète un grand nombre de pièces: Diamorphoses (1957), Concret PH (1958), Analogique B (1959), Orient-Occident (1960), Bohor (1962)... Ceci dit, Xenakis aura des relations assez tendues avec Pierre Schaeffer, comprenant d'une manière très personnelle l'idéologie de la musique concrète que celui-ci tentait de formaliser. ${ }^{6}$ Il n'en reste pas moins que cette expérience du studio de musique électroacoustique sera déterminante.

\footnotetext{
Olivier Messiaen, cité dans: Nouritza Matossian, Iannis Xenakis, Paris, Fayard/Sacem, 1981, p. 90.

Cf. l'article de François Delalande et Évelyne Gayou, "Xenkis et le GRM", in Présences de Iannis Xenakis, sous la direction de Makis Solomos, Paris, CDMC, 2001.
} 
C'est aussi en 1954, quand il vient à Paris travailler au studio pour la création de Déserts, la première pièce pour orchestre et bande stéréophonique, que Xenakis fait la connaissance d'Edgar Varèse. Varèse est évidemment plus âgé que Xenakis, mais c'est pourtant un des compositeurs les plus visionnaire. Nouritza Matossian fait remarquer à juste titre à quel point on peut lire dans cet extrait d'une conférence de Varèse datant de 1936 des phrases qui sont quasiment prémonitoires de l'esthétique de Xenakis.

"Ces zones (d'intensité) seraient différenciées par divers timbres ou couleurs et différents volumes sonores. Grâce à un tel procédé matériel, ces zones paraîtraient de couleurs et d'ampleur différentes selon les perspectives de notre perception. Le rôle de la couleur ou du timbre serait complètement changé du fait d'être accidentel, anecdotique, sensuel ou pittoresque; il deviendrait un agent de délimitation de la même façon que les diverses couleurs d'une carte géographique séparent diverses zones, et le nonmélange, jusqu'ici impossible à obtenir, deviendrait possible.

Dans les volumes en mouvement l'on prendrait conscience de leur transmutations lorsqu'ils passent au-dessus de différentes couches, lorsqu'ils pénètrent certaines opacités, ou se dilatent au sein de certaines raréfactions." ${ }^{7}$

Un grand intérêt pour l'espace, pour le mouvement, mais aussi une attirance certaine pour le surgissement d'une énergie primordiale, liée au son lui-même, ou au rythme dans son expression la plus physique, relient la musique de Xenakis à celle de Varèse. On peut aussi probablement remonter dans cette direction à une des œuvres qui a révélé à la musique son potentiel de sauvage modernité: Le sacre du printemps, d'Igor Stravinsky, dont on sait que Xenakis a écouté l'analyse dans la classe de Messiaen, et dont on ne peut pas s'empêcher de retrouver un écho dans les passages de Jonchaie(1977) où un martèlement binaire, très appuyé, vient secouer l'orchestre et les auditeurs sans ménagement.

Le Groupe de Recherches Musicale n'est pas pour Xenakis qu'une occasion de confrontation avec des aînés. C'est aussi l'endroit, après la classe de Messiaen, où il pourra briser son isolement de créateur et trouver des compagnons de route. François Bernard Mâche sera probablement un des plus proches de lui, d'abord parce qu'il est à même, ayant fait des études de littérature classique très avancées, de comprendre le fond de culture grecque qui irrigue la pensée de Xenakis, et puis aussi parce qu'il est traversé comme lui par un questionnement fondamental concernant le rapport de la musique aux modèles naturels. Il ne faut pas oublier que l'on est en France, dans les années soixante, en pleine période structuraliste. Boulez cite Levi-Strauss. Xenakis, pour sa part, se démarque très clairement de toute tentative de considérer la musique comme un langage, et de la soumettre à l'aune de la linguistique. Elle doit répondre selon lui à une catégorisation plus originelle, une paramétrisation plus physique et philosophique.

"Je ne crois pas qu'aucune tentative de considérer la musique comme un langage puisse aboutir. L'infrastructure d'une musique est beaucoup plus proche de l'espace et du temps. La musique est plus pure, beaucoup plus proche des catégories de l'esprit.. ${ }^{8}$

Au delà des querelles de techniciens qui habitaient l'époque sérielle, la pensée musicale de Xenakis désigne un autre projet, un projet qui met la musique au centre de préoccupations qui concernent l'esprit et la pensée, depuis la source mythique jusqu'à la

\footnotetext{
Edgar Varèse, cité dans: Nouritza Matossian, Iannis Xenakis, Paris, Fayard/Sacem, 1981, p. 91. Iannis Xenakis, cité dans: Nouritza Matossian, Iannis Xenakis, Paris, Fayard/Sacem, 1981, p. 109.
} 
métaphore spatiale du cosmos. François Bernard Mâche reprend cette idée en la développant dans un ouvrage intitulé Musique, Mythe, Nature:

"Depuis Debussy, en passant par le premier Stravinsky, par certaines œuvres de Messiaen, de Bartók et de Prokofiev, et surtout par Varèse et par Xenakis, toute une part de la création musicale occidentale n'est concernée essentiellement ni par les problèmes de forme ni par les soucis de la communication, mais par la recherche d'une nouvelle finalité musicale. [...] La musique n'est pas une sorte de langage, elle est une pensée plus générale, plus proche de la source mythique (mythifiante, plus exactement) [...] La seule crise musicale profonde de notre époque concerne le pourquoi, et non le comment de la musique. L'ère laïque du pseudo-'langage' musical soi-disant autonome me paraît révolue. La musique n'est plus 'le seul système signifiant privé de référent', comme on l'a trop dit. Cette pureté a toujours été illusoire, et désormais les sons réels l'ont submergée de matériaux nouveaux ; elle a mieux à faire qu'à se soucier d'en créer d'autres. Mais pour savoir qu'en faire, il existe depuis Debussy une route précise: la création musicale pourrait être pour l'essentiel la rencontre entre les archétypes et une phénoménologie du monde sonore, entre l'inné et le perçu.."

Et plus loin il ajoute:

"Chercher des modèles dans la nature n'est pas se chercher des contraintes par peur de la liberté, c'est chercher l'usage le plus efficace de la liberté, la mesure de cette efficacité étant la joie, dont la conquête est une mission de la musique, et qui ne fait qu'un avec le pouvoir sur soi et sur les choses." ${ }^{10}$

On est bien loin d'une conception matérialiste et industrieuse de l'usage des mathématiques en musique, conception à laquelle on a voulu parfois un peu trop rapidement réduire l'auteur de Musiques formelles. Certes Xenakis va puiser dans l'abstraction symbolique des statistiques, de la théorie des groupes ou encore dans les opérations algorithmiques, des outils utiles et efficaces pour écrire sa musique. Certes, il se sert de ce paravent théorique pour ne pas mettre en avant les aspects plus intimes de son œuvre. Mais cela ne doit pas nous leurrer sur la profondeur de ses intentions, une profondeur éthique qui guide, bien avant sa reconnaissance comme compositeur, son comportement d'homme et de citoyen, une profondeur éclairée par ce sens aigu de la liberté que décrit François Bernard Mâche. D’ailleurs, Xenakis écrit lui-même au sujet de la pensée et de l'enseignement de Messiaen:

"Ce que j'apprenais là, c'était la liberté qu'un compositeur aurait pu avoir, sans les œillières des écoles ou des styles, sans les conventions sociales." ${ }^{11}$

Cet amour de la liberté va jusqu'à lui faire exprimer une certaine défiance vis à vis de la science elle-même:

"Si nous voulons combattre cette tendance fondamentale [l'obéissance], nous devons nous méfier des idéologies et de leurs incarnations institutionnelles, telles que les fabriquent les partis. Nous devons nous méfier même des théories scientifiques, que ce

9 François-Bernard Mâche, Musique, mythe, nature, Paris, Klincksieck, 1983, p. 60.

10 Id. p. 114.

11 Iannis Xenakis, "Ouvrir les fenêtres sur l'inédit", in 20ème siècle, images de la musique française, Jean-Pierre Derien ed., Paris, Sacem \& Papiers, 1986, p. 160. 
soit en physique, en mathématiques, en astrophysique, en biologie ; nous devons nous méfier comme de la peste de toute chose qui semble établie et vraie.. ${ }^{12}$

S'il est capable d'exiger cet esprit critique des rapports avec l'outil scientifique, c'est aussi pour combattre haut et fort, et aussi par les voies de l'art, un certain nombre de problèmes liés à la société. Xenakis restera un éternel résistant:

"Un questionnement perpétuel devrait être à la base de l'exercice de la liberté ; c'est peut-être dans le domaine artistique que cet exercice se développe de la manière la plus riche. Je suis musicien, et je sais que l'art est le domaine le plus propice à la liberté d'expression. [...] l'art est, à mon avis, plus libre que la science. Mais dans le domaine artistique en Occident, et ailleurs également, des institutions empêchent la véritable liberté de pensée et d'expression artistiques. Ici, par exemple, les conservatoires constituent de véritables camps retranchés, parce que des professeurs dont la formation est exclusivement conservatrice se révèlent trop souvent limités et parce qu'une conception élitiste de l'art, généralement répandue, réserve à quelques-uns seulement ce qui devrait être mis à la portée de tous. Il faut que l'exercice de la création appartienne aux masses. Ces propos ne relèvent pas de la démagogie, mais tenir ce langage, c'est dire qu'on retrouve chez tout homme l'étincelle divine de Dionysos, qui lui permet d'agir et de comprendre. Si la majorité des gens n'étaient pas sensibles aux phénomènes artistiques, il n'y aurait jamais eu d'art du tout.

Enfin, dans les pays de civilisation ancienne, comme l'Europe, les structures de la pensée sont archaïques et sclérosées, le poids du passé joue de façon décisive en faveur de l'esclavage et de la domination. Ce phénomène est sensible à la radio et à la télévision, qui sont au service de goûts momifiés et ignorent la pensée actuelle. Il ne s'agit pas de détruire le passé et les traditions, comme j'en parlais plus haut, mais de comprendre que la tradition figée est dangereuse, tandis que la tradition rénovée par un esprit bien vivant peut être une source de richesses extraordinaires. Le passé, si l'on se contente de le subir, condamne une civilisation à l'asphyxie progressive. Prenons-y garde. Il existe un seuil de la création en deçà duquel on ne peut plus créer, et c'est au niveau de l'individu, de la famille, de la tribu, de l'État que se manifestent les conséquences culturelles. Je crains fort que nous n'en soyons là en Europe, dans des pays qui maintiennent obstinément leurs frontières et leurs chauvinismes de clocher. Un passé prestigieux peut créer des inhibitions qui anéantiront tout effort de renouvellement." ${ }^{13}$

Je cite ce passage de manière un peu extensive car il me paraît fondamental pour bien comprendre un aspect très important de la personnalité de Xenakis et qui mérite d'autant plus aujourd'hui d'être mis en avant: son engagement politique et moral. On peut difficilement comprendre la musique de Xenakis sans aborder cette thématique. Certaines œuvres comme Nuits ou À l'île de Gorée y font explicitement référence.

Aussi, le formalisme et l'utilisation de l'informatique, dont il a été un des pionniers, ne doivent pas être compris chez lui, ce qui parfois été le cas, comme un asservissement à la logique scientifique dominante, mais bien comme la recherche d'outils pour libérer l'écoute du poids de la tradition musicale. Autour du CEMAMu, qu'il fonde en 1966, puis

12 Iannis Xenakis, Kéleztha, Paris, l'arche, 1994, p. 133. (le texte original date de 1984).

13 Ibid. p. 134-135. 
de la réalisation de l'UPIC, il fédérera un nombre important de chercheurs et de musiciens concernés par une nouvelle manière de penser le musical. Parmi ceux-ci, on peut noter André Riotte, qui avec Marcel Mesnage, mettra au point le Morphoscope, un outil informatique pour analyser les formes musicales, et qui ira jusqu'au bout des implications concrètes de la notion de modélisation en musique.

De 1972 à 1989, Xenakis enseigne à l'Université de Paris I Sorbonne, Université dont il sera reçu docteur en 1976. Ce sera l'occasion pour lui de fréquenter des musiciens plus jeunes. Mais ses cours ne sont pas des cours de composition comme on l'entend communément. Xenakis refuse une quelconque autorité dans un domaine où il cherche à promouvoir au contraire la recherche personnelle et la libre créativité de chacun. Il transmet ses connaissances, montre ses recherches, discute, quand on l'y provoque, de ses conceptions. Mais il ne donnera pas d'avis sur la musique des autres. Pascal Dusapin fréquente les cours de Xenakis de 1974 à 1978. Il est indéniable que la musique de Xenakis aura une influence déterminante sur la sienne, influence que l'on peut entendre directement par exemple dans le premier trio à corde, ou dans Khôra. On peut lire dans la biographie de Dusapin publiée par l'IRCAM: '[Xenakis] le réconnaît volontiers comme son seul élève: 'J'aime Pascal Dusapin parce qu'il est fier, curieux, indépendant et organisé dans sa pensée" Cela n'empêchera pas Dusapin de se démarquer de son influence, et d'instruire, même si c'est avec révérence, le procès fait par les compositeurs de sa génération contre Xenakis. Le premier trouble naît du rapport art-science, revendiqué avec vigueur mais réalisé avec parfois peu de rigueur par Xenakis.

"Sa pensée, profondément pénétrée par l'idée pythagoricienne de l"Art/Science' a cependant engendré les pires confusions. Si le formalisme de Xenakis commence par le modèle mathématique, c'est que son esprit s'exalte sans cesse à l'idée d'une forme absolue d'une solidité structurelle sans faille. Mais, dès que l'analyse de ses oeuvres en révèle les détours, les fuites même devant la rectitude de sa méthode, il peut apparaître alors comme un 'léger mystique'.

N'en concluons pas qu'il s'agit avec lui d'un scientisme impur, d'un écran scintillant et trompeur; l'apprentissage solitaire et douloureux de cette approche plurale de l'art fut son premier outil formel. Elle lui permet une imagination "au-dessus" des normes de la technique musicale ou de celles des "intégristes de la pure raison". [...]

Sa musique a effectivement opéré une catalyse avec la science, mais n'a jamais pu créer un axiome qui puisse fondamentalement valider la thèse de l'„Art/Sciencen. L'originalité de sa démarche, bien qu'elle ait permis de solutionner certains aspects de l'alternative musicale des années 50/60, reste néanmoins une théorie de l'analogie et du transfert de modèle.

La question est de savoir à présent si l'incertaine relation entre les sciences et les arts formulée par Xenakis sera un facteur d'évolutions et de mutations pour notre conscience, ou si elle ne restera que la fabuleuse coïncidence d'un homme parvenu aux plus hauts degrés de la création, grâce à l'originalité de sa formation physique et spirituelle." ${ }^{14}$

Pour Dusapin, renvoyer Xenakis à n'être qu'un épiphénomène, c'est aussi chercher à remettre la bonne vieille conscience musicale en première ligne. Même si Xenakis

14 Pascal Dusapin, "L'imagination au-dessus", Festival d'Automne à Paris 1972-1982, Jean-Pierre Leonardini, Marie Collin et Joséphine Markovits Ed., Paris Messidor/Temps Actuels, 1982, p. 217-218. 
propose des chemins inouïs, notre écoute a ses attentes, et ce sont elles qui dominent notre perception des œuvres, quoi qu'en décrètent les équations. C'est en substance ce qu'il confie à Harry Halbreich en juin 1981:

"Là où Xenakis pèche un peu, c'est que de toute manière notre écoute est orientée, et que, l'audition d'une œuvre comme Phithoprakta, il y a des intervalles qui dominent instantanément, et des lignes mélodiques qui ressortent de la masse. [... ${ }^{15}$

Il poursuit:

"Il y a un problème peut-être beaucoup plus important et qui caractérise toute cette génération de musiciens. Rétrospectivement, lorsque je me souviens des propos de Xenakis à ses cours, je m'aperçois qu'il ne parlait jamais de grandes formes [...] Il s'agissait toujours d'une analyse ponctuelle." ${ }^{16}$

Il faut sérieusement nuancer le sous-entendu de Dusapin concernant la conscience que Xenakis avait de la grande forme. Toute analyse de ses œuvres prouve à quel point il était un des compositeurs de sa génération le plus au clair avec les problèmes de construction musicale à grande échelle. Il tenait peut-être cette capacité de son métier d'architecte. En tout cas, il y a peu de musiques aussi équilibrées, aussi soucieuses de la trajectoire dynamique que celle de Xenakis. Quant à son obsession du ponctuel pendant les cours, je n'ai certes pas suivi les mêmes que Pascal Dusapin ${ }^{17}$, mais j'ai le net souvenir que la réponse qu'il m'avait faîte concernant justement ce problème de la forme en musique était une des plus lumineuse qu'il m'ait été donné d'entendre, à une époque où régnait, en particulier dans l'orbite sérielle, une confusion assez flagrante sur ce sujet.

Ceci dit, il est clair que dans les années quatre-vingt, la musique réinvestit l'aspect perceptif, et cela vaut aussi pour Xenakis. Un des archétypes de ce phénomène concerne la réapparition de notes polaires, qui servent d'axe de référence pour l'écoute. C'est ce que Xenakis appellera les isons. On trouve cette pratique, contraire à la déontologie de non-répétition et d' 'atonalité' qui prévalait dans la période précédente (à l'exception notable de quelques œuvres de Ligeti et de Scelsi), chez presque tous les compositeurs de l'époque, même chez Boulez. C'est évidemment particulièrement flagrant chez Dusapin dans son opéra To be sung où l'avant dernière section qui s'intitule Chapters when they need a monk, par exemple, met en place un bourdon avec des voix qui psalmodient à l'unisson ou à l'octave. On trouve également cette nécessité dans l'écriture de Félix Ibarrondo, par exemple dans son trio à cordes Phalène, bien que d'une manière plus souple et plus dramatisée. Mais au delà de cet aspect particulier, il y a la claire conscience que la musique doit revenir au ventre de sa matrice sonore originelle. Le spectralisme est sans doute une des formes de cette régression, régression nécessaire sans doute pour découvrir d'autres enjeux musicaux.

Les deux déclarations qui suivent, respectivement de Dusapin et de Grisey, explicitent assez bien la défiance qui règne sur le rôle que doit jouer l'extra-musicalité dans la pensée musicale:

"Depuis quelque temps, il y a une chose que j'essaie absolument d'éviter, malgré

15 Pascal Dusapin, In Regards sur Iannis Xenakis op. cit. p. 347.

16 Id.

17 L'auteur de cet article a suivi la dernière année de cours de Xenakis à Paris I. 
mon amour pour sa musique, c'est son aspect symbolique, en tant que le son chez Xenakis, et notamment dans de très grandes œuvres comme Herma ou Eonta, est la représentation d'une entité abstraite, complètement imaginaireé... philosophique." ${ }^{18}$

"Nous sommes des musiciens et notre modèle, c'est le son, non la littérature, le son, non les mathématiques, le son, non le théâtre, les arts plastiques, la théorie des quanta, la géologie, l'astrologie ou l'acupuncture." ${ }^{19}$

Questionné par Jean-Pierre Derien sur ses rapports avec la musique française, Xenakis raconte comment en décembre 1944, pendant l'occupation de la Grèce par les Anglais, il a découvert la musique française sous les doigts d'un compagnon de combat. 'À l'écoute de Debussy et de Ravel, j'ai immédiatement pensé que c'était, là, la musique qui correspondait le mieux à la pensée antique, peut-être surtout par le climat général qui s'en dégage... Le Tombeau de Couperin ne m'apparaissait pas comme une référence au XVII. siècle, mais comme un véritable tombeau antique. Je découvrais un monde que j'avais déjà en moi, le mien...'

à r. composé en 1987, en hommage à Maurice Ravel (à l'occasion du cinquantenaire de sa mort) est la seule pièce où Xenakis fait explicitement référence à Ravel. Pourtant, elle est issue, comme la quasi-totalité des pièces de cette période de la théorie des cribles. L'arpégiation ondulée qui la traverse, martelée par quelques accords, n'est pas sans évoquer un autre hommage à Ravel, celui que Grisey lui rend dans Vortex temporum. Comment ne pas noter, dans les deux cas, la présence de la figure de l'onde, et celle du geste circulaire qui décrit l'harmonie par l'égrenage d'une échelle/accord ? Au fond, quelle que soit l'étendue de ses explorations, un musicien n'oublie jamais qu'il est d'abord musicien, et il faut peut-être faire entendre ici ces phrases attribuées à Ravel lui-même: 'Je n'ai jamais éprouvé le besoin de formuler, soit pour autrui, soit pour moi-même, les principes de mon esthétique. Si j'étais tenu de le faire, je demanderais la permission de reprendre à mon compte les simples déclarations que Mozart a faites à ce sujet. Il se bornait à dire que la musique peut tout entreprendre, tout oser et tout peindre, pourvu qu'elle charme et reste enfin et toujours la musique. On s'est plu, parfois, à me prêter des opinions, fort paradoxales en apparence, sur le mensonge de l'art et les dangers de la sincérité. Le fait est que je me refuse simplement mais absolument à confondre la conscience de l'artiste, qui est une chose, avec sa sincérité, qui en est une autre. La seconde n'est d'aucun prix si la première ne l'aide pas à se manifester. Cette conscience exige que nous développions en nous le bon ouvrier. Mon objectif est donc la perfection technique. Je puis y tendre sans cesse, puisque je suis assuré de ne jamais l'atteindre. L'important est d'en approcher toujours davantage. L'art, sans doute, a d'autres effets, mais l'artiste, à mon gré, ne doit pas avoir d'autre but. ${ }^{20}$

Id. p. 354 .

9 Gérard Grisey, "La musique: le devenir des sons", in Darmstädter Beiträge zur Neuen Musik, vol. XIX, Mainz, 1984, p. 22.

20 Rapporté par Roland-Manuel, in Marcel Marnat, Maurice Ravel, Qui êtes-vous? Paris, La Manufacture 1987, p. 63. 